\title{
CBF changes and cerebral energy metabolism during hypervolemia, hemodilution, and hypertension therapy in patients with poor-grade subarachnoid hemorrhage
}

\author{
Henrik Engquist, MD, ${ }^{1,2}$ Anders Lewén, MD, PhD, ${ }^{1}$ Lars Hillered, MD, PhD, ${ }^{1}$ \\ Elisabeth Ronne-Engström, MD, PhD, ${ }^{1}$ Pelle Nilsson, MD, PhD, ${ }^{1}$ Per Enblad, MD, PhD, ${ }^{1}$ and \\ Elham Rostami, MD, PhD' \\ Departments of ${ }^{1}$ Neuroscience/Neurosurgery and ${ }^{2}$ Surgical Sciences/Anesthesia and Intensive Care, Uppsala University, \\ Uppsala, Sweden
}

OBJECTIVE Despite the multifactorial pathogenesis of delayed cerebral ischemia (DCI) after subarachnoid hemorrhage (SAH), augmentation of cerebral blood flow (CBF) is still considered essential in the clinical management of $\mathrm{DCl}$. The aim of this prospective observational study was to investigate cerebral metabolic changes in relation to CBF during therapeutic hypervolemia, hemodilution, and hypertension $(\mathrm{HHH})$ therapy in poor-grade $\mathrm{SAH}$ patients with $\mathrm{DCl}$.

METHODS CBF was assessed by bedside xenon-enhanced CT at days $0-3,4-7$, and 8-12, and the cerebral metabolic state by cerebral microdialysis (CMD), analyzing glucose, lactate, pyruvate, and glutamate hourly. At clinical suspicion of $\mathrm{DCl}, \mathrm{HHH}$ therapy was instituted for 5 days. $\mathrm{CBF}$ measurements and $\mathrm{CMD}$ data at baseline and during $\mathrm{HHH}$ therapy were required for study inclusion. Non-DCl patients with measurements in corresponding time windows were included as a reference group.

RESULTS In DCI patients receiving HHH therapy $(n=12)$, global cortical CBF increased from $30.4 \mathrm{ml} / 100 \mathrm{~g} / \mathrm{min}$ (IQR $25.1-33.8 \mathrm{ml} / 100 \mathrm{~g} / \mathrm{min}$ ) to $38.4 \mathrm{ml} / 100 \mathrm{~g} / \mathrm{min}$ (IQR $34.2-46.1 \mathrm{ml} / 100 \mathrm{~g} / \mathrm{min} ; \mathrm{p}=0.006$ ). The energy metabolic CMD parameters stayed statistically unchanged with a lactate/pyruvate (L/P) ratio of 26.9 (IQR 22.9-48.5) at baseline and 31.6 (IQR 22.4-35.7) during HHH. Categorized by energy metabolic patterns during HHH, no patient had severe ischemia, 8 showed derangement corresponding to mitochondrial dysfunction, and 4 were normal. The reference group of non-DCl patients $(n=11)$ had higher CBF and lower L/P ratios at baseline with no change over time, and the metabolic pattern was normal in all these patients.

CONCLUSIONS Global and regional CBF improved and the cerebral energy metabolic CMD parameters stayed statistically unchanged during $\mathrm{HHH}$ therapy in $\mathrm{DCl}$ patients. None of the patients developed metabolic signs of severe ischemia, but a disturbed energy metabolic pattern was a common occurrence, possibly explained by mitochondrial dysfunction despite improved microcirculation.

https://thejns.org/doi/abs/10.3171/2019.11.JNS192759

KEYWORDS subarachnoid hemorrhage; delayed cerebral ischemia; cerebral blood flow; hypertension, hypervolemia, and hemodilution therapy; triple-H; xenon CT; cerebral microdialysis; vascular disorders

$\mathrm{D}$ ELAYED cerebral ischemia (DCI) is a common complication in the acute course of severe subarachnoid hemorrhage (SAH) and contributes substantially to mortality and long-term morbidity in this group of patients. ${ }^{6,33}$ The modern understanding of DCI is that the causes are multifactorial, with vasospasm as one of many contributing factors. The process starts already at the aneurysm rupture and ictus of intracranial hypertension, causing global ischemia and early brain injury, and is then further escalated by degradation products from the clot, inflammatory response, microthrombosis, and microcirculatory disturbances. ${ }^{18}$ Aside from nimodipine, no spe-

ABBREVIATIONS $\mathrm{ACA}=$ anterior cerebral artery; $\mathrm{CBF}=$ cerebral blood flow; $\mathrm{CMD}=$ cerebral microdialysis; $\mathrm{CPP}=$ cerebral perfusion pressure; $\mathrm{DCl}=$ delayed cerebral ischemia; GCS = Glasgow Coma Scale; HHH = hypervolemia, hemodilution, and hypertension; ICP = intracranial pressure; L/P = lactate/pyruvate; MAP = mean arterial pressure; $\mathrm{MCA}=$ middle cerebral artery; $\mathrm{NIC}=$ neurosurgical intensive care; $\mathrm{PCA}=$ posterior cerebral artery; $\mathrm{rCBF}=$ regional $\mathrm{CBF} ; \mathrm{ROI}=$ region of interest; $\mathrm{SAH}=$ subarachnoid hemorrhage; $\mathrm{SBP}=$ systolic blood pressure; $\mathrm{TBI}=$ traumatic brain injury; $\mathrm{XeCT}=$ xenon-enhanced $\mathrm{CT}$.

SUBMITTED October 10, 2019. ACCEPTED November 5, 2019.

INCLUDE WHEN CITING Published online January 10, 2020; DOI: 10.3171/2019.11.JNS192759. 
cific pharmacological treatment has proven to reduce the incidence of DCI or improve outcome. ${ }^{2}$

Compromised cerebral blood flow (CBF) may, by itself, contribute to the progression of DCI, and augmentation of $\mathrm{CBF}$ is considered essential in the management of patients suspected of having DCI. ${ }^{4,33}$ The concept of hemodynamic augmentation of CBF originally included hypervolemia, hemodilution, and hypertension $(\mathrm{HHH})$ therapy to improve systemic blood flow and cerebral perfusion pressure (CPP), and to optimize blood rheology ${ }^{15,23}$ Prophylactic use of HHH has been abandoned as the side effects might worsen outcome. ${ }^{8,17}$ However, it is established that ensuring normovolemia and normotension reduces the risk of DCI.$^{19,20}$ Therapeutic use of HHH at suspicion of DCI has been addressed in several small and uncontrolled studies, but the results are divergent and there is no clear evidence concerning the effects of the different elements of HHH. ${ }^{5}$ Most guidelines now recommend only blood pressure augmentation, which is still based on weak scientific evidence. .,32 $^{3}$

When DCI is suspected in patients with SAH at our unit, our standardized neurosurgical intensive care (NIC) protocol includes careful application of $\mathrm{HHH}$ therapy with a moderately increased blood pressure target and vigilant hemodynamic reevaluation to minimize side effects. In a previous observational study at our institution, using xenon-enhanced CT (XeCT), CBF increased during $\mathrm{HHH}$ therapy, ${ }^{9}$ but there are still uncertainties regarding whether this leads to increased oxygen delivery in cerebral regions at risk and restoration of cellular energy metabolism. To our knowledge, there are few studies combining repeated measurement of $\mathrm{CBF}$ and continuous monitoring of cerebral energy metabolism in poor-grade SAH patients. ${ }^{24,29}$ Therefore, additional studies on the effects of therapeutic interventions to resolve DCI in these patients may provide valuable information.

The aim of this prospective observational study was to investigate cerebral metabolic changes in relation to $\mathrm{CBF}$ during $\mathrm{HHH}$ therapy at suspicion of DCI in patients with poor-grade SAH. CBF was assessed by bedside XeCT and the cerebral metabolic state by cerebral microdialysis (CMD). The primary hypothesis was that the cerebral metabolic state improves as CBF increases during $\mathrm{HHH}$ therapy, i.e., a less ischemic CMD pattern with decreased lactate level and lactate/pyruvate $(\mathrm{L} / \mathrm{P})$ ratio.

\section{Methods \\ Patients}

The study was conducted in a cohort of patients with severe spontaneous SAH, verified by CT, who had CBF measured by XeCT during their early acute course in NIC at Uppsala University Hospital from 2013 to 2016. All patients were on mechanical ventilation at the time of inclusion due to their initial level of consciousness or early neurological deterioration. $\mathrm{CBF}$ measurement using $\mathrm{XeCT}$ in our setting is only performed in intubated patients, which was thus a criterion for inclusion. Criteria for exclusion were pronounced intracranial hypertension, deep barbiturate sedation, inspired oxygen demand $>60 \%$, inability to obtain consent from the patient's next of kin, and decisions to withhold treatment. The study protocol was approved by the Uppsala University Regional Ethical Review Board and informed consent was obtained from the patients included or their next of kin. The study was also approved by the local radiation safety authority.

\section{Study Design}

Following our clinical NIC routine, intubated SAH patients should have XeCT CBF measurements at days $0-3$, $4-7$, and $8-12$ if logistically possible. For the present study of cerebral energy metabolism in relation to CBF during $\mathrm{HHH}$ therapy, patients who had been examined with $\mathrm{XeCT}$ and who had a CMD catheter inserted at the same procedure as ventriculostomy were included. Data from CBF measurements and CMD, as well as clinical and physiological data, were prospectively collected. The neurological state of the patients was repeatedly evaluated. If the patient was determined to have a clinical diagnosis of DCI, HHH therapy was initiated in accordance with our standard NIC protocol as described below. To study the effects of HHH therapy, a baseline measurement of CBF was required within 48 hours before the start of $\mathrm{HHH}$, and a second measurement was required during the 5-day course of the therapy. As a reference group, patients with $\mathrm{CBF}$ measurements at corresponding time windows but with no suspicion of DCI were identified.

\section{$\mathrm{XeCT}$ for Measurement of CBF}

In our NIC unit, assessment of CBF in mechanically ventilated patients is provided by bedside $\mathrm{XeCT}$, following the principles originally developed by Gur et al. and Yonas et al. ${ }^{11,34}$ The inert xenon gas dissolves readily in blood and tissues, and acts as a radiographic contrast agent due to its high atomic number. Hence, inhaled nonradioactive xenon serves as a diffusible tracer during a repeated series of 4 axial CT scans and allows CBF to be calculated based on the Fick principle as applied by Kety for inert gas uptake in tissue. ${ }^{16}$ Xenon (28\% in air/oxygen) is administered to the patients' breathing circuit by a computer-controlled delivery system (Enhancer 3000, Diversified Diagnostic Products Inc.), while synchronized CT scans are acquired by a mobile CT scanner (CereTom, Neurologica). CBF in each CT pixel is calculated by the integrated software, and finally mean blood flow is routinely calculated for each of 20 regions of interest (ROIs) defined in the CT images at three scan levels (Fig. 1A). ROIs in areas of hematoma or containing radiological artifacts are manually excluded.

\section{Calculated CBF Parameters From XeCT}

Global cortical CBF (ml/100 g/min) was calculated as the mean (weighted by ROI size) of all ROIs at all scan levels, typically a total of 60 ROIs. Regional CBF of the worst vascular territory (rCBF worst, $\mathrm{ml} / 100 \mathrm{~g} / \mathrm{min}$ ) was identified for each patient and calculated as the mean of the corresponding cortical ROIs at all scan levels: anterior cerebral artery (ACA), middle cerebral artery (MCA), and posterior cerebral artery (PCA) territories of the right and left hemisphere, respectively (Fig. 1A).

To quantify the distribution of areas with critically low and near-ischemic blood flow, thresholds for local CBF 

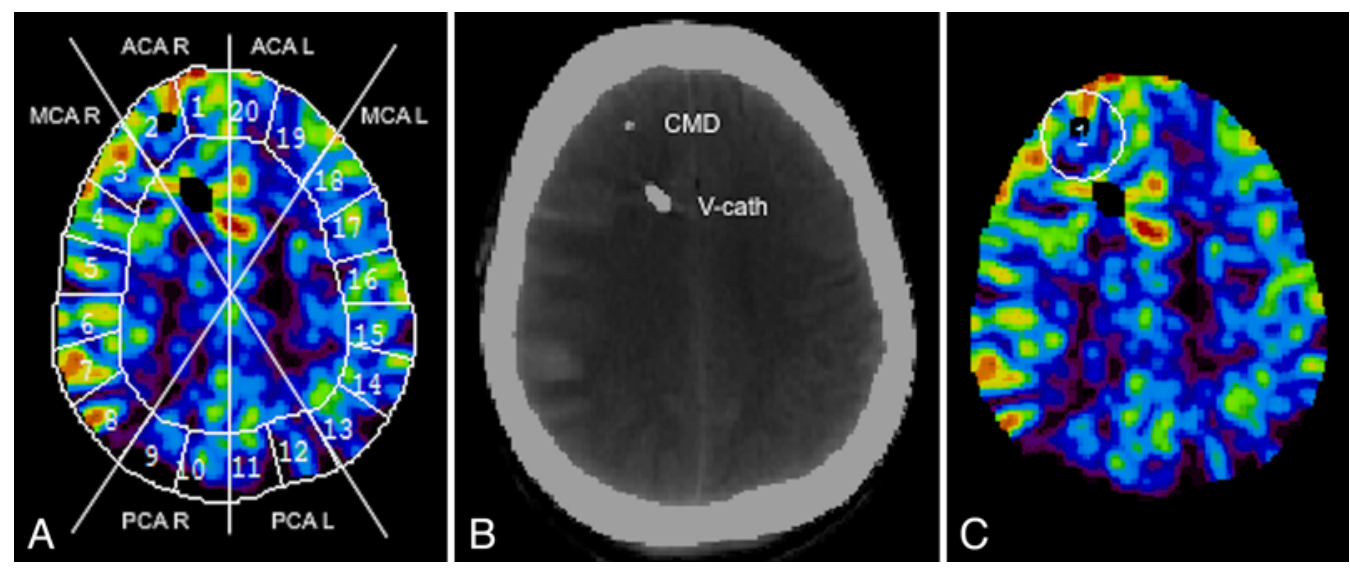

FIG. 1. Example of an XeCT image with 20 cortical ROls symmetrically set out (A), and an ROI set at the CMD catheter area (C, circle). The CMD ROI area was $509 \mathrm{~mm}^{2}$, and the $\mathrm{rCBF}$ was $35.9 \mathrm{ml} / 100 \mathrm{~g} / \mathrm{min}$. The ventriculostomy catheter is also visible in the CT image (V-cath; B). The main vascular territories were approximated to the corresponding ROls in each hemisphere.

were set to 10 and $20 \mathrm{ml} / 100 \mathrm{~g} / \mathrm{min}^{1,10}$ The proportion of cortical ROI area with local CBF below the specified thresholds was calculated as the sum of ROI area $\left(\mathrm{mm}^{2}\right)$ with local CBF below the threshold divided by the total analyzed ROI area in each patient. Regional $\mathrm{CBF}$ of the CMD catheter ROI area (rCBF CMD, ml/100 g/min; Fig. 1B) was calculated from an ROI (approximately $500 \mathrm{~mm}^{2}$ ) manually set out in the XeCT image at the location of the tip of the CMD catheter (Fig. 1C).

\section{Cerebral Microdialysis}

The technique for CMD is well established in NIC for assessment of the metabolic state of the brain by measurement of interstitial glucose, lactate, pyruvate, and glutamate equilibrated to a microdialysis perfusion fluid circulated through a semipermeable microcatheter. ${ }^{12,13,21}$ As a routine clinical procedure in patients with severe $\mathrm{SAH}$, a CMD catheter is placed in the cortex of the right frontal lobe at the same procedure as the insertion of a ventriculostomy catheter. For logistic and technical reasons, not all intubated SAH patients received CMD catheters. The CMD catheter used was the 70 Brain Microdialysis catheter (M Dialysis AB), with a 20-kDa cutoff membrane of 10-mm length. Artificial "cerebrospinal fluid" was perfused through the catheter at a rate of $0.3 \mu \mathrm{l} / \mathrm{min}$ using a microinjection pump (CMA-106, M Dialysis AB). The fluid had a composition of $\mathrm{NaCl} 147 \mathrm{mmol} / \mathrm{L}, \mathrm{KCl}$ $2.7 \mathrm{mmol} / \mathrm{L}, \mathrm{CaCl}_{2} 1.2 \mathrm{mmol} / \mathrm{L}$, and $\mathrm{MgCl}_{2} 0.85 \mathrm{mmol} / \mathrm{L}$ (Perfusion Fluid CNS, M Dialysis AB). Hourly CMD samples were collected and analyzed at bedside using the CMA600 or the ISCUSflex Microdialysis Analyzer (M Dialysis AB). The level of CMD urea was monitored for validation of catheter performance. ${ }^{25}$

\section{CMD Parameters and Classification of CMD Metabolic Patterns}

For this study, the means of CMD measurements (glucose, lactate, pyruvate, and glutamate) for 2 hours before and 2 hours after the XeCT CBF measurements were used. The L/P ratio was calculated for each CMD sample. In a consensus report from 2015, critical levels of these CMD parameters were defined as glucose $<0.2-0.8 \mathrm{mmol} / \mathrm{L}$, lactate $>4 \mathrm{mmol} / \mathrm{L}$, and $\mathrm{L} / \mathrm{P}$ ratio $>25-40 .^{13}$

Additionally, the consensus report states that a pattern of increased $\mathrm{L} / \mathrm{P}$ ratio and low pyruvate (in combination with low brain tissue oxygen if available) suggests an ischemic energy crisis, whereas a pattern of increased $\mathrm{L} / \mathrm{P}$ ratio and normal/high pyruvate (in combination with normal brain tissue oxygen if available) suggests a nonischemic energy crisis (e.g., mitochondrial dysfunction) ${ }^{13}$ In two recent studies on NIC patients with severe $\mathrm{SAH}^{14}$ and severe traumatic brain injury (TBI), ${ }^{22}$ cerebral ischemia was defined as an L/P ratio $>30$ and pyruvate $<70$ $\mu \mathrm{mol} / \mathrm{L}$, and cerebral mitochondrial dysfunction as an $\mathrm{L} / \mathrm{P}$ ratio $>30$ and pyruvate $>70 \mu \mathrm{mol} / \mathrm{L}$. Based on these statements and studies, three patterns of the CMD parameters were defined to reflect the cerebral energy metabolic state of each patient in this study: normal (lactate $<4 \mathrm{mmol} / \mathrm{L}$, $\mathrm{L} / \mathrm{P}$ ratio $<30)$, mitochondrial dysfunction $(\mathrm{L} / \mathrm{P}$ ratio $>$ 30 , pyruvate $>70 \mu \mathrm{mol} / \mathrm{L}$ ), or ischemia $(\mathrm{L} / \mathrm{P}$ ratio $>30$, pyruvate $<70 \mu \mathrm{mol} / \mathrm{L}$ ).

Finally, CMD glutamate was used as a biomarker of excitotoxicity, implicated as a secondary injury mechanism in SAH. ${ }^{13}$ The critical level of CMD glutamate was considered to be $>15 \mu \mathrm{mol} / \mathrm{L}$, based on a previous SAH study. ${ }^{31}$

\section{NIC of SAH Patients}

SAH patients admitted to our unit are managed in accordance with a standardized NIC protocol for SAH, as described by Ryttlefors et al., 27 including multimodal monitoring of physiological and biochemical parameters and vigilance for avoidable factors to minimize secondary brain injury. Sedation is titrated with propofol (Fresenius Kabi AB) 0-4 mg/kg/hr and morphine (Meda AB) as needed. In patients with altered levels of consciousness or hydrocephalus, an external ventricular drain is placed for intracranial pressure (ICP) monitoring and CSF drainage. ICP exceeding $20 \mathrm{~mm} \mathrm{Hg}$ is treated with open ventricular drainage set at a pressure level of $15 \mathrm{~mm} \mathrm{Hg}$. Aneurysms are, as a rule, treated early, preferably with endovascular coil embolization when feasible, or with surgical clipping 
TABLE 1. Characteristics for the group with a clinical diagnosis of $\mathrm{DCl}$ who subsequently received $\mathrm{HHH}$ therapy, and the reference group (non-DCl patients) with $\mathrm{CBF}$ measurements at corresponding time windows

\begin{tabular}{|c|c|c|}
\hline Characteristic & $\begin{array}{c}\mathrm{DCl} \\
\text { (HHH therapy) }\end{array}$ & $\begin{array}{l}\text { No } \mathrm{DCl} \\
\text { (ref group) }\end{array}$ \\
\hline No. of patients & 12 & 11 \\
\hline Female/males & $10 / 2$ & $9 / 2$ \\
\hline Mean age, yrs (range) & $62(48-75)$ & $58(28-84)$ \\
\hline \multicolumn{3}{|l|}{ Treatment modality } \\
\hline Endovascular & 12 & 7 \\
\hline Surgical clip & 0 & 4 \\
\hline \multicolumn{3}{|l|}{ CT Fisher grade } \\
\hline 3 & 5 & 2 \\
\hline 4 & 7 & 9 \\
\hline \multicolumn{3}{|l|}{ Hunt \& Hess grade } \\
\hline \multicolumn{3}{|l|}{ At admission } \\
\hline$|-|||$ & 8 & 3 \\
\hline IV-V & 4 & 8 \\
\hline \multicolumn{3}{|l|}{ At baseline $\mathrm{XeCT}$} \\
\hline III & 3 & 4 \\
\hline IV-V & 9 & 7 \\
\hline \multicolumn{3}{|c|}{$\begin{array}{l}\text { Clinical neurological course at NIC } \\
\text { discharge }\end{array}$} \\
\hline GCS motor score 6 & 7 & 9 \\
\hline GCS motor score $\leq 5$ & 5 & $2^{*}$ \\
\hline \multicolumn{3}{|c|}{ Infarct $>20 \mathrm{~mm}$ at $\mathrm{CT}$, day $\geq 12$} \\
\hline Yes & 2 & 4 \\
\hline No & 10 & 7 \\
\hline
\end{tabular}

Ref = reference.

* One patient in the reference group died on day 11 in the NIC.

if indicated. The target for systemic volume status is normovolemia to mild hypervolemia if not contraindicated by cerebral edema or hematoma with mass effect and/or elevated ICP. To ensure volume status, fluids are administrated in the higher normal range and patients are monitored by central venous pressure, clinical evaluation, and attentive fluid balance calculation. Albumin infusion (200 $\mathrm{mg} / \mathrm{ml}$, Baxter AG) is used for further volume expansion if needed. Mean arterial pressure (MAP) is kept above 85 $\mathrm{mm} \mathrm{Hg}$, and if vasoactive support is required, dobutamine (Hospira) is used as first-line and norepinephrine (Hospira Nordic AB) as second-line treatment. Nimodipine (Bayer Pharma $A G$ ) is routinely given from admission onward.

\section{Diagnosis of $\mathrm{DCl}$ and HHH Therapy}

The patients with SAH are repeatedly evaluated for neurological deterioration, and in cases of new focal deficits or reduced levels of consciousness, DCI is clinically diagnosed if other causes for deterioration are ruled out. In addition to standard therapy, HHH therapy to augment $\mathrm{CBF}$ is then initiated for 5 days with daily infusions of 500 $\mathrm{ml}$ dextran-40 solution (100 mg/ml, Meda AB) and 200 $\mathrm{ml}$ albumin $(200 \mathrm{mg} / \mathrm{ml})$. During HHH therapy, the pa- tient is kept supine and the focus is on ensuring adequate intravascular volume status, while the blood pressure target is moderately elevated with a lower limit for systolic blood pressure (SBP) of $140 \mathrm{~mm} \mathrm{Hg}$. As described above, the first choice of vasoactive support is dobutamine and/or the addition of norepinephrine if needed. The neurological and hemodynamic situation is repeatedly reevaluated to minimize the risk of serious side effects.

\section{Statistical Methods}

SPSS statistical software (version 23.0, IBM Corp.) was used for statistical analyses of the collected data. Differences in systemic physiological parameters between measurements were tested by paired samples t-test. CBF and CMD data for groups of patients are presented as median values and IQR because of non-normal distribution. Differences in CBF and CMD parameters between related samples were tested by Wilcoxon signed-ranks test, and between independent samples by Mann-Whitney U-test. The statistical significance level was set at $\mathrm{p}<0.05$.

\section{Results}

Twenty-four patients clinically diagnosed with DCI during their course in the NIC had appropriate CBF measurements at the defined time windows both prior to the start of HHH therapy and during the therapy. Among these HHH-treated patients, 12 also had CMD data, including the time points for XeCT procedures. In the nonDCI reference group with measurements in corresponding time windows, CMD data were available for 11 patients. Clinical characteristics of these patients are presented in Table 1.

The median time point for initiation of HHH therapy was 3.1 days (IQR 1.9-4.5 days) from admission to NIC. For DCI patients, the median time for the baseline XeCT from admission was 2.0 days (IQR 1.5-4.3 days) and for the examination during HHH therapy 6.6 days (IQR 5.27.6 days). The median time for the reference (non-DCI) patients with $\mathrm{XeCT}$ in the corresponding time windows was 2.8 days (IQR 2.0-3.0 days) and 5.4 days (IQR 4.7-5.9 days; Fig. 2), respectively.

\section{Systemic Physiological Conditions}

SBP and MAP, CPP, arterial $\mathrm{PaCO}_{2}$, body temperature, and hematocrit at the start of the XeCT measurements at baseline and at the second time window are presented for the respective groups in Table 2 (lower). During $\mathrm{HHH}$ therapy, the mean SBP for the HHH-treated DCI group was increased from $152.9 \mathrm{~mm} \mathrm{Hg}$ (95\% CI 141.5-164.3 $\mathrm{mm} \mathrm{Hg}$ ) at baseline to $160.7 \mathrm{~mm} \mathrm{Hg}(95 \%$ CI 151.6-169.9 $\mathrm{mm} \mathrm{Hg}$ ), but this increase was not statistically significant. In the DCI group there was a moderate decrease in hematocrit from $34.9 \%$ (95\% CI $33.2 \%-36.6 \%$ ) at baseline to $30.6 \%$ (95\% CI $29.0 \%-32.2 \%$; $\mathrm{p}=0.001$ ). Dobutamine and norepinephrine were used in only a few patients and at low doses; the number of patients and dose ranges are shown in Table 2. In the reference group, modest elevations of $\mathrm{PaCO}_{2}$ and body temperature between the two measurements were noted. No significant differences were found for these parameters in the DCI group (Table 2). 


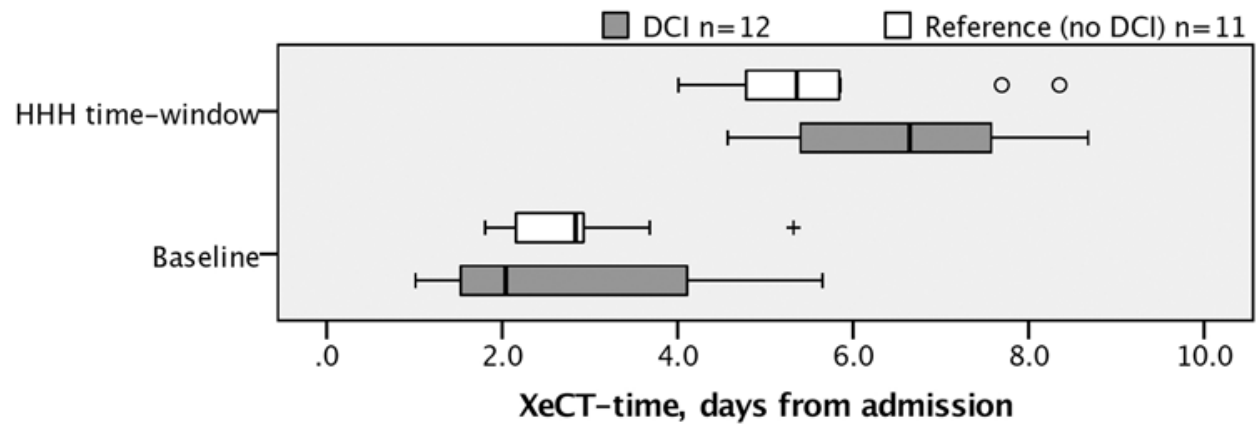

FIG. 2. Median time (days) from admission to the baseline XeCT CBF measurement and to the measurement during HHH therapy for $\mathrm{DCl}$ patients (gray), and time to the corresponding measurements for the reference group (non-DCl patients; white). Circles and + sign indicate values exceeding 1.5 times the IQR.

\section{CBF at Baseline}

Global cortical CBF at baseline among patients subsequently diagnosed with DCI was markedly lower than for patients in the reference group: median $30.4 \mathrm{ml} / 100 \mathrm{~g} / \mathrm{min}$ (IQR 25.1-33.8 ml/100 g/min) compared with $40.1 \mathrm{ml} / 100$ $\mathrm{g} / \mathrm{min}$ (IQR 31.5-60.1 ml/100 g/min; $\mathrm{p}=0.026$; Fig. 3A). There were also differences in $\mathrm{rCBF}$ parameters at baseline (Fig. 3B and C). Median rCBF of the worst vascular territory in the DCI group was $19.6 \mathrm{ml} / 100 \mathrm{~g} / \mathrm{min}$ (IQR $12.1-26.5 \mathrm{ml} / 100 \mathrm{~g} / \mathrm{min}$ ) compared with $30.4 \mathrm{ml} / 100 \mathrm{~g} /$ $\mathrm{min}$ (IQR $21.4-53.2 \mathrm{ml} / 100 \mathrm{~g} / \mathrm{min}$ ) in the reference group $(\mathrm{p}=0.005)$. The proportion of low-flow ROI area (local $\mathrm{CBF}<20 \mathrm{ml} / 100 \mathrm{~g} / \mathrm{min}$ ) was a median of $27.7 \%$ (IQR $9.8 \%-39.4 \%)$ compared with $6.7 \%(1.7 \%-16.7 \%$; $\mathrm{p}=$ $0.007)$ in the reference group.

\section{Changes in Global and rCBF During HHH Therapy}

The results of the CBF measurements at baseline and during $\mathrm{HHH}$ therapy and corresponding measurements for the reference group are presented in Table 2 and Fig. 3 . For patients receiving HHH therapy, there was a significant increase in global cortical CBF from $30.4 \mathrm{ml} / 100 \mathrm{~g} /$ $\min$ (IQR $25.1-33.8 \mathrm{ml} / 100 \mathrm{~g} / \mathrm{min}$ ) to $38.4 \mathrm{ml} / 100 \mathrm{~g} / \mathrm{min}$ (IQR 34.2-46.1 ml/100 g/min; $p=0.006$; Fig. 3A). A similar pattern was seen for rCBF during HHH therapy with an increase in $\mathrm{rCBF}$ of the worst vascular territory from a median of $19.6 \mathrm{ml} / 100 \mathrm{~g} / \mathrm{min}$ (IQR $12.1-26.5 \mathrm{ml} / 100 \mathrm{~g} /$ $\mathrm{min}$ ) to $31.5 \mathrm{ml} / 100 \mathrm{~g} / \mathrm{min}$ (IQR $22.1-38.5 \mathrm{ml} / 100 \mathrm{~g} / \mathrm{min}$; $\mathrm{p}=0.005)$ and a decrease in proportion of low-flow ROI area (local $\mathrm{CBF}<20 \mathrm{ml} / 100 \mathrm{~g} / \mathrm{min}$ ) from a median of $27.7 \%(9.8 \%-39.4 \%)$ to $7.2 \%$ (2.0\%-23.0\%; $\mathrm{p}=0.019$; Fig. $3 \mathrm{~B}$ and $\mathrm{C})$.

Concerning the reference (non-DCI) group, a small decrease in CBF between the two measurements was statistically nonsignificant, and the regional parameters remained at an unchanged level at the second time window.

\section{Regional CBF in Proximity to the CMD Catheter Versus Global Cortical CBF}

At the baseline measurement, the worst vascular territory was found ipsilateral to the location of the CMD catheter in 4 of the 12 DCI patients and in 4 of the 11 reference patients. Regional CBF of the ROI set in the area near the tip of the CMD catheter followed the same pattern as the global $\mathrm{CBF}$ and $\mathrm{rCBF}$ for both DCI and reference patients (Fig. 3D). In the DCI group, rCBF CMD increased from $25.6 \mathrm{ml} / 100 \mathrm{~g} / \mathrm{min}$ (IQR $22.5-35.9 \mathrm{ml} / 100 \mathrm{~g} / \mathrm{min}$ ) to 37.4 $\mathrm{ml} / 100 \mathrm{~g} / \mathrm{min}$ (IQR $30.2-46.5 \mathrm{ml} / 100 \mathrm{~g} / \mathrm{min} ; \mathrm{p}=0.004$ ). Based on all XeCT measurements conducted in the 23 patients, there was a positive correlation between global cortical CBF and $\mathrm{rCBF}$ of the CMD ROI (Spearman correlation $r_{s}=0.68, p<0.001$; Fig. 4).

\section{Metabolic CMD Parameters at Baseline}

Data for the metabolic CMD parameters are presented in conjunction with CBF parameters in Table 2 and Fig. 3E-H. CMD lactate at baseline for patients subsequently diagnosed with DCI was $4.37 \mathrm{mmol} / \mathrm{L}$ (IQR 3.41-5.58 $\mathrm{mmol} / \mathrm{L}$ ) compared to $2.42 \mathrm{mmol} / \mathrm{L}$ (IQR 2.13-2.97 $\mathrm{mmol} / \mathrm{L} ; \mathrm{p}=0.026$ ) for non-DCI patients in the reference group. The difference in CMD pyruvate between the groups at baseline did not reach statistical significance $(162.0 \mu \mathrm{mol} / \mathrm{L}$ [IQR $113.2-179.1 \mu \mathrm{mol} / \mathrm{L}]$ vs $117.5 \mu \mathrm{mol} / \mathrm{L}$ [IQR 85.7-174.1 $\mu \mathrm{mol} / \mathrm{L}])$. The L/P ratio was higher in the DCI group: 26.9 (IQR 22.9-48.5) versus 20.3 (IQR 18.8 vs $26.2 ; \mathrm{p}=0.044)$. There was no significant difference in baseline CMD glucose between the groups.

\section{Metabolic CMD Parameters During HHH Therapy in DCI Patients}

During $\mathrm{HHH}$ therapy there was no significant change in CMD lactate from the baseline level $(4.37 \mathrm{mmol} / \mathrm{L}$ [IQR 3.41-5.58 $\mathrm{mmol} / \mathrm{L}$ ] at baseline vs $4.78 \mathrm{mmol} / \mathrm{L}$ [IQR 3.71-5.15 $\mathrm{mmol} / \mathrm{L}]$ during HHH). CMD pyruvate and the $\mathrm{L} / \mathrm{P}$ ratio also stayed statistically unchanged at the same levels as in the baseline measurements (Table 2, Fig. 3F$\mathrm{H})$. Similarly, no change was noted in the CMD glucose level for the DCI group.

\section{Metabolic CMD Parameters Over Time for the Reference Group}

For the non-DCI patients in the reference group, a slight decrease was detected in CMD glucose from 2.52 $\mathrm{mmol} / \mathrm{L}$ (IQR $1.27-2.87 \mathrm{mmol} / \mathrm{L}$ ) at baseline to 1.77 $\mathrm{mmol} / \mathrm{L}(\mathrm{IQR} 0.74-2.60 \mathrm{mmol} / \mathrm{L}$ ) at the day 5-8 time window $(\mathrm{p}=0.050)$. There was also a small increase in CMD lactate from $2.42 \mathrm{mmol} / \mathrm{L}$ (IQR 2.13-2.97 mmol/L) 


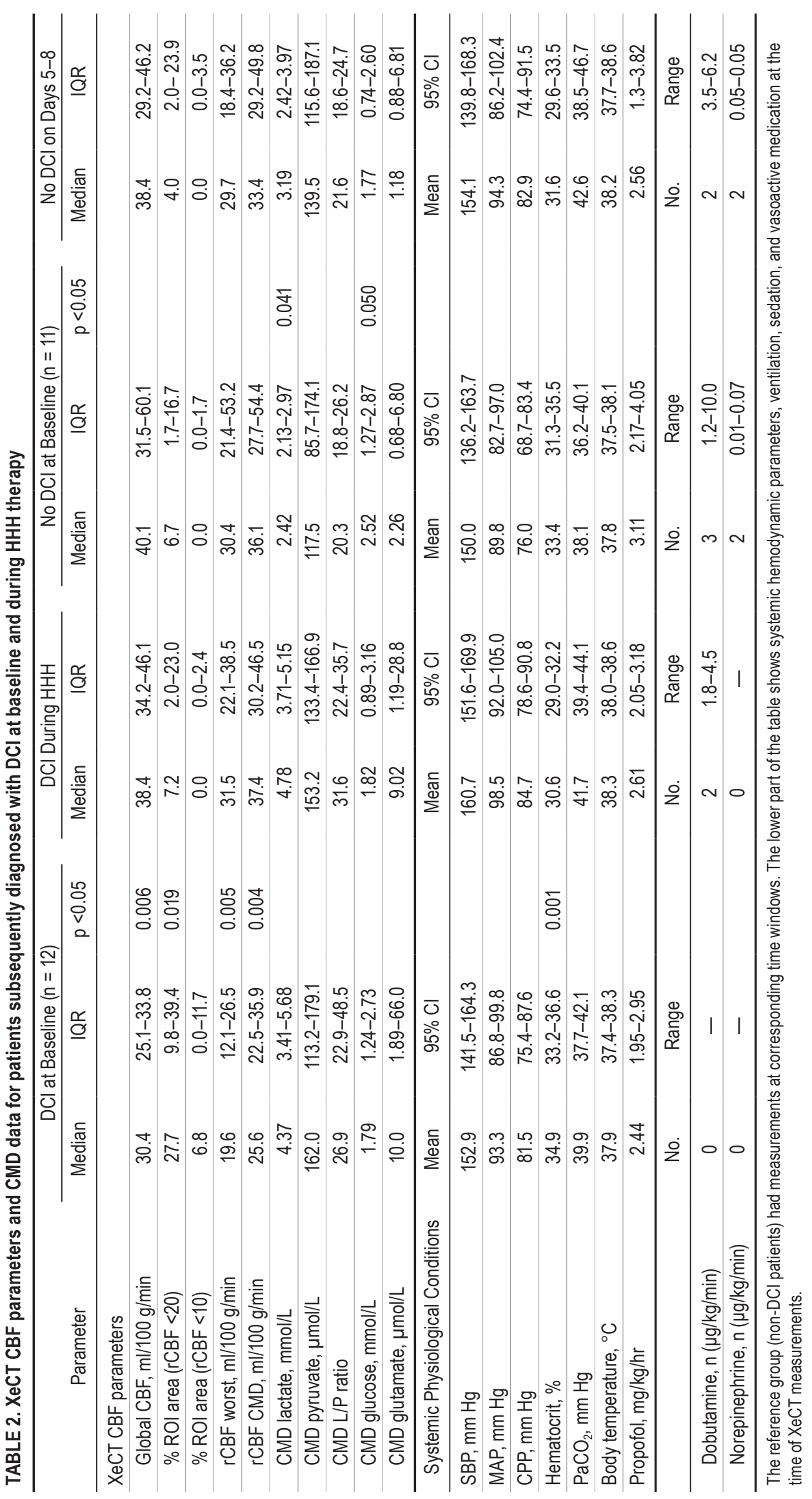



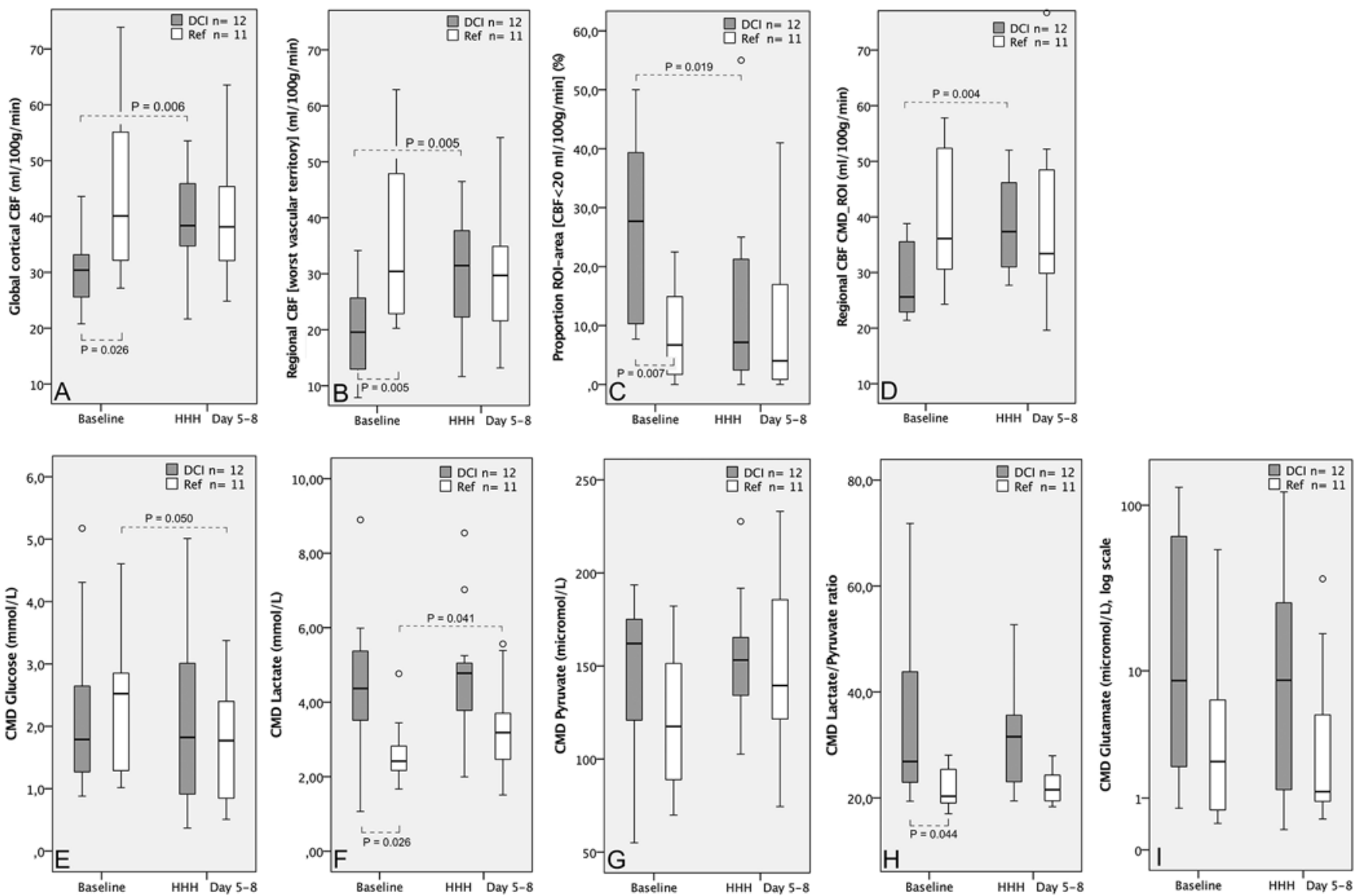

FIG. 3. Box plots (median, IQR) of CBF parameters (A-D) and cerebral microdialysis parameters $(E-I)$ at baseline and during $\mathrm{HHH}$ therapy for patients diagnosed with $\mathrm{DCl}$ (gray) or at day 5-8 for the reference group (white). A: Global cortical CBF. B: Regional $\mathrm{CBF}$ of worst vascular territory. C: Proportion of ROI area with CBF $<20 \mathrm{ml} / 100 \mathrm{~g} / \mathrm{min}$. D: Regional CBF of CMD ROI. E: CMD glucose. F: CMD lactate. G: CMD pyruvate. H: CMD L/P ratio. I: CMD glutamate.

to $3.19 \mathrm{mmol} / \mathrm{L}$ (IQR $2.42-3.97 \mathrm{mmol} / \mathrm{L} ; \mathrm{p}=0.041$ ), while pyruvate and the $\mathrm{L} / \mathrm{P}$ ratio stayed statistically unchanged.

\section{Patients Categorized by CMD Energy Metabolic Patterns at the Two CBF Measurements}

When the classification of three different CMD patterns (normal, mitochondrial dysfunction, or ischemia) was applied to the DCI group at baseline, there were 4 patients in mitochondrial dysfunction, 1 in ischemia, and 7 were normal (Fig. 5). At the measurement during $\mathrm{HHH}$ therapy, no patient showed ischemia, 8 patients were categorized with a pattern of mitochondrial dysfunction, and 4 were normal. In the reference group of non-DCI patients, all were categorized as normal both at baseline and at the second time window (Fig. 5).

\section{Excitotoxicity: CMD Glutamate}

At baseline, median CMD glutamate for the DCI group was $10.0 \mu \mathrm{mol} / \mathrm{L}$ (IQR $1.89-66.0 \mu \mathrm{mol} / \mathrm{L}$ ) compared to $2.26 \mu \mathrm{mol} / \mathrm{L}$ (IQR $0.68-6.80 \mu \mathrm{mol} / \mathrm{L}$ ) for the reference group, but the difference did not reach statistical significance $(p=0.065$; Table 2, Fig. 3I). There was no significant change from baseline to the second measurement for either of the groups. In the DCI group, CMD glutamate exceeding $15 \mu \mathrm{mol} / \mathrm{L}$ was noted in 6 patients at baseline and in 4 during $\mathrm{HHH}$, suggesting excitotoxicity, whereas only 1 patient in the reference group had CMD glutamate above this threshold.

\section{Clinical Course and Infarcts at NIC Discharge}

At discharge from the NIC, 7 (58\%) of 12 patients in the DCI group and $9(82 \%)$ of 11 patients in the reference group had a Glasgow Coma Scale (GCS) motor score of 6 (Table 1). One of the patients in the reference group died on day 11 in the NIC. Infarcts $>20 \mathrm{~mm}$ at follow-up CT (day 12 or later) were found in 2 patients in the DCI group $(17 \%)$ and 4 patients (36\%) in the reference group.

\section{Discussion}

The standardized protocol for SAH in our unit includes cautious application of HHH therapy with focus on ensuring adequate intravascular filling and keeping a moderate blood pressure target, as described in the Methods section. In a previously published study on the effect of $\mathrm{HHH}$ therapy in patients with poor-grade $\mathrm{SAH}$ in our unit, an increase in CBF during treatment was concluded..$^{9}$ The intention with the present study was to investigate whether 


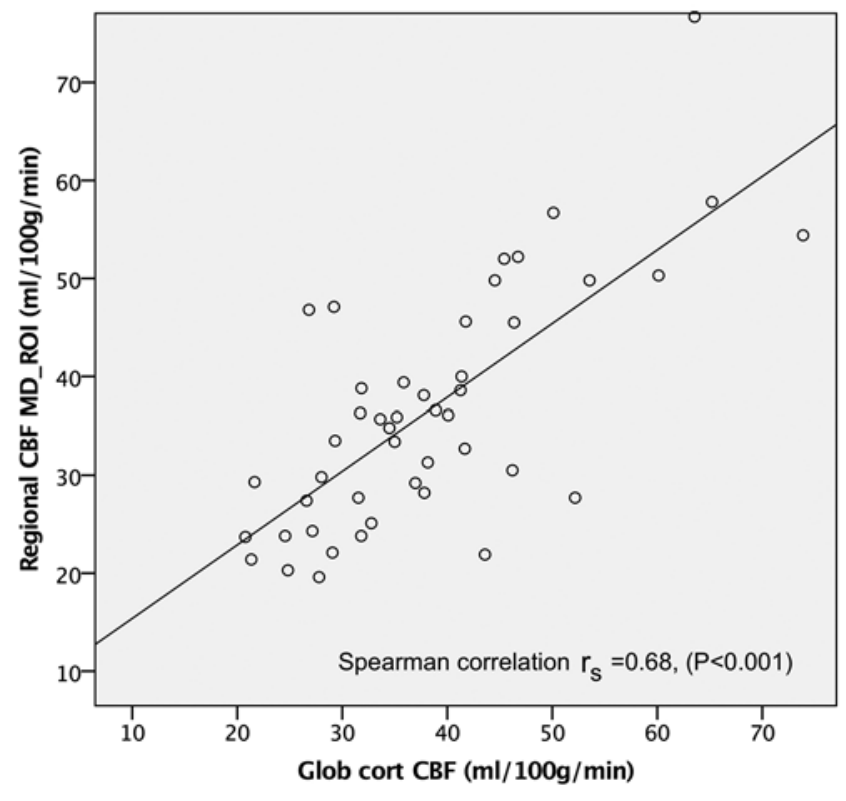

FIG. 4. Scatterplot and regression line of global cortical CBF and rCBF at the area adjacent to the CMD catheter based on all XeCT measurements conducted in the 23 patients ( $\mathrm{DCl}$ and reference group). There was a positive correlation between global cortical $\mathrm{CBF}$ and $\mathrm{rCBF}$ of the CMD catheter ROI area; Spearman correlation $r_{s}=0.68, p<0.001$.

the energy metabolic pattern, as measured by interstitial $\mathrm{CMD}$, changes in concordance with the improvement in CBF during HHH therapy.

The patients who later developed DCI had markedly lower global cortical CBF at baseline compared to patients in the reference group, which is consistent with other studies. ${ }^{3,28} \mathrm{CBF}$ of the worst vascular territory and the proportion of ROI area with near-ischemic flow showed corresponding differences. Regarding the CMD parameters, there was a significantly higher lactate level in the DCI group at baseline, and there was also a higher $\mathrm{L} / \mathrm{P}$ ratio for this group. It appears plausible that the pathological metabolic state at baseline reflects the situation with compromised $\mathrm{CBF}$ in the DCI patients, as has been demonstrated in earlier studies. ${ }^{21,24}$ When the DCI patients were categorized by energy metabolic CMD patterns, only 1 of the DCI patients was found in ischemia at baseline and 4 in mitochondrial dysfunction, indicating that the energy metabolic derangement was moderate in the majority of these patients.

At the measurement during $\mathrm{HHH}$ therapy in the DCI group, there was an increase in both global cortical CBF and $\mathrm{rCBF}$ of the worst vascular region. There was also a marked reduction in the proportion of ROI area with nearischemic flow. However, the CMD lactate level as well as pyruvate and the L/P ratio stayed statistically unchanged during HHH. Following clinical diagnosis of DCI and the start of HHH therapy, the proportion of patients with an energy metabolic CMD pattern of mitochondrial dysfunction increased from 4 to 8 , and 4 remained in a normal CMD pattern. Previous CMD studies similarly found ischemia to be a much less common type of energy crisis than mitochondrial dysfunction in both SAH and TBI

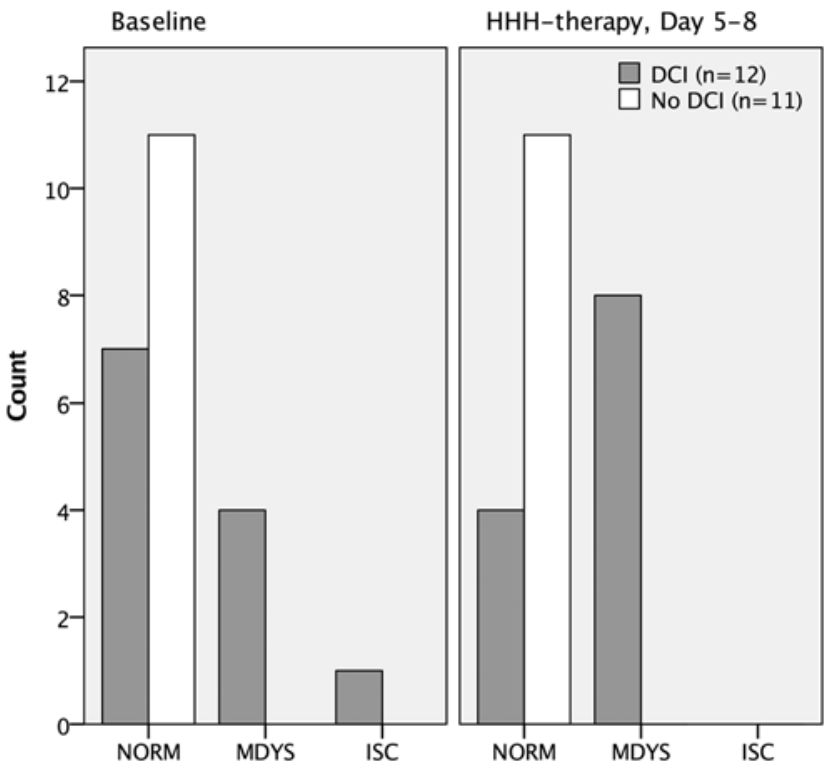

FIG. 5. Patients categorized by three cerebral microdialysis patterns at baseline and during $\mathrm{HHH}$ therapy for the $\mathrm{DCl}$ group (gray) or at day 5-8 for the reference group (white). ISC $=$ ischemia (L/P ratio $>30$, pyruvate $<70 \mu \mathrm{mol} / \mathrm{L})$; MDYS = mitochondrial dysfunction (L/P ratio $>30$, pyruvate $>70 \mu \mathrm{mol} / \mathrm{L}) ; \mathrm{NORM}=$ normal $($ lactate $<4 \mathrm{mmol} / \mathrm{L}, \mathrm{L} / \mathrm{P}$ ratio $<30)$.

patients..$^{14,22}$ Our results are also consistent with a previous study on SAH patients showing no significant effect of $\mathrm{HHH}$ therapy on CMD glucose, lactate, or $\mathrm{L} / \mathrm{P}$ ratio in DCI patients despite clinical neurological improvement. ${ }^{30}$ The value of these observations is limited due to the low number of patients in our study, but suggests that the HHH therapy may have been beneficial, however with a common occurrence of disturbed energy metabolism despite improved CBF. As the mode of HHH in our standard protocol is cautious, it cannot be ruled out that more aggressive hemodynamic therapy would show different results.

Among the patients in the reference group, a small decrease in global cortical CBF at the second time window did not reach statistical significance, and the regional parameters remained unchanged. There was also a small, statistically significant increase in CMD lactate in these patients, but pyruvate and the $\mathrm{L} / \mathrm{P}$ ratio were unchanged, all at a lower level compared to the DCI group. These findings are consistent with the reference group having less affected CBF and no obvious clinical signs of DCI.

Regarding excitotoxicity, an increase in CMD glutamate has been associated with neurological deterioration and ischemic changes in SAH patients. ${ }^{29}$ Although the trend was a higher glutamate level in the DCI group at baseline, the range was wide and the difference between the groups did not reach statistical significance. The glutamate level stayed unchanged for both groups at the second measurement. These data support the findings of the energy metabolic patterns, but are partly in contrast to the previously mentioned CMD study in which the glutamate level decreased after HHH therapy. ${ }^{30}$

No specific analysis of outcome was attempted due to the relatively small group of patients with SAH studied. To reflect the clinical course, the GCS motor score and pres- 
ence of infarcts at NIC discharge were determined. The picture was somewhat ambiguous, as the reference group had a numerically higher proportion of patients with favorable GCS motor score, but fewer infarcts were detected in the DCI group.

\section{Limitations}

There are some inherent limitations in this observational study. First, there is no valid control group, as all patients with clinical signs of DCI received HHH therapy following our standard protocol. The non-DCI patients, referred to as a reference group, may have differences in the pathophysiology of cerebral hemodynamics and energy metabolism that make comparisons inconclusive. Second, the number of patients receiving $\mathrm{HHH}$ therapy, who also had $\mathrm{CBF}$ measurements in adequate time windows and had functioning CMD throughout the treatment course, was limited. The low number of patients makes generalization of our findings uncertain, and large differences are required to obtain statistical significance. A third shortcoming is from the method of CMD, reflecting the metabolic situation in a rather small region of the brain. This limits the ability of CMD to detect localized or scattered disturbances in the cerebral circulation. There was, however, a good correlation between global $\mathrm{CBF}$ and $\mathrm{rCBF}$ in the CMD region, which has also been demonstrated in previous studies. ${ }^{26}$

\section{Conclusions}

The main findings of this study are that global and rCBF improved and the cerebral energy metabolic CMD parameters stayed statistically unchanged during HHH therapy in DCI patients. None of the patients developed metabolic signs of severe ischemia, but a disturbed energy metabolic pattern was a common occurrence, possibly explained by mitochondrial dysfunction despite a proposed beneficial effect from $\mathrm{HHH}$ on the cerebral microcirculation. Our findings emphasize the importance of hemodynamic management and multimodal monitoring in poor-grade $\mathrm{SAH}$ patients to optimize $\mathrm{CBF}$, but also imply that other factors impairing the cerebral energy metabolism at the cellular level should be addressed in future research.

\section{Acknowledgments}

The excellent work of Johan Bäckander and Caroline Elofsson in performing $\mathrm{XeCT}$ was greatly appreciated.

\section{References}

1. Baron JC: Perfusion thresholds in human cerebral ischemia: historical perspective and therapeutic implications. Cerebrovasc Dis 11 (Suppl 1):2-8, 2001

2. Brathwaite $\mathrm{S}$, Macdonald RL: Current management of delayed cerebral ischemia: update from results of recent clinical trials. Transl Stroke Res 5:207-226, 2014

3. Dankbaar JW, de Rooij NK, Rijsdijk M, Velthuis BK, Frijns CJ, Rinkel GJ, et al: Diagnostic threshold values of cerebral perfusion measured with computed tomography for delayed cerebral ischemia after aneurysmal subarachnoid hemorrhage. Stroke 41:1927-1932, 2010

4. Dankbaar JW, de Rooij NK, Smit EJ, Velthuis BK, Frijns CJ, Rinkel GJ, et al: Changes in cerebral perfusion around the time of delayed cerebral ischemia in subarachnoid hemorrhage patients. Cerebrovasc Dis 32:133-140, 2011

5. Dankbaar JW, Slooter AJ, Rinkel GJ, Schaaf IC: Effect of different components of triple-H therapy on cerebral perfusion in patients with aneurysmal subarachnoid haemorrhage: a systematic review. Crit Care 14:R23, 2010

6. Dhar R, Diringer MN: Relationship between angiographic vasospasm, cerebral blood flow, and cerebral infarction after subarachnoid hemorrhage. Acta Neurochir Suppl 120:161165,2015

7. Diringer MN, Bleck TP, Claude Hemphill J III, Menon D, Shutter L, Vespa P, et al: Critical care management of patients following aneurysmal subarachnoid hemorrhage: recommendations from the Neurocritical Care Society's Multidisciplinary Consensus Conference. Neurocrit Care 15:211-240, 2011

8. Diringer MN, Zazulia AR: Aneurysmal subarachnoid hemorrhage: strategies for preventing vasospasm in the intensive care unit. Semin Respir Crit Care Med 38:760-767, 2017

9. Engquist H, Rostami E, Ronne-Engström E, Nilsson P, Lewén A, Enblad P: Effect of HHH-therapy on regional CBF after severe subarachnoid hemorrhage studied by bedside xenon-enhanced CT. Neurocrit Care 28:143-151, 2018

10. Fainardi E, Tagliaferri MF, Compagnone C, Tanfani A, Cocciolo F, Battaglia R, et al: Regional cerebral blood flow levels as measured by xenon-CT in vascular territorial low-density areas after subarachnoid hemorrhage are not always ischemic. Neuroradiology 48:685-690, 2006

11. Gur D, Good WF, Wolfson SK Jr, Yonas H, Shabason L: In vivo mapping of local cerebral blood flow by xenon-enhanced computed tomography. Science 215:1267-1268, 1982

12. Hillered L, Persson L, Pontén U, Ungerstedt U: Neurometabolic monitoring of the ischaemic human brain using microdialysis. Acta Neurochir (Wien) 102:91-97, 1990

13. Hutchinson PJ, Jalloh I, Helmy A, Carpenter KL, Rostami E, Bellander BM, et al: Consensus statement from the 2014 International Microdialysis Forum. Intensive Care Med 41:1517-1528, 2015

14. Jacobsen A, Nielsen TH, Nilsson O, Schalén W, Nordström $\mathrm{CH}$ : Bedside diagnosis of mitochondrial dysfunction in aneurysmal subarachnoid hemorrhage. Acta Neurol Scand 130:156-163, 2014

15. Kassell NF, Peerless SJ, Durward QJ, Beck DW, Drake CG, Adams HP: Treatment of ischemic deficits from vasospasm with intravascular volume expansion and induced arterial hypertension. Neurosurgery 11:337-343, 1982

16. Kety SS: The measurement of cerebral blood flow by means of inert diffusible tracers. Keio J Med 43:9-14, 1994

17. Lennihan L, Mayer SA, Fink ME, Beckford A, Paik MC, Zhang H, et al: Effect of hypervolemic therapy on cerebral blood flow after subarachnoid hemorrhage: a randomized controlled trial. Stroke 31:383-391, 2000

18. Macdonald RL: Delayed neurological deterioration after subarachnoid haemorrhage. Nat Rev Neurol 10:44-58, 2014

19. Maroon JC, Nelson PB: Hypovolemia in patients with subarachnoid hemorrhage: therapeutic implications. Neurosurgery 4:223-226, 1979

20. Nakagawa A, Su CC, Sato K, Shirane R: Evaluation of changes in circulating blood volume during acute and very acute stages of subarachnoid hemorrhage: implications for the management of hypovolemia. J Neurosurg 97:268-271, 2002

21. Nilsson OG, Brandt L, Ungerstedt U, Säveland H: Bedside detection of brain ischemia using intracerebral microdialysis: subarachnoid hemorrhage and delayed ischemic deterioration. Neurosurgery 45:1176-1185, 1999

22. Nordström CH, Nielsen TH, Schalén W, Reinstrup P, Ungerstedt U: Biochemical indications of cerebral ischaemia and mitochondrial dysfunction in severe brain trauma anal- 
ysed with regard to type of lesion. Acta Neurochir (Wien) 158:1231-1240, 2016

23. Origitano TC, Wascher TM, Reichman OH, Anderson DE: Sustained increased cerebral blood flow with prophylactic hypertensive hypervolemic hemodilution ("triple-H" therapy) after subarachnoid hemorrhage. Neurosurgery 27:729-740, 1990

24. Patet C, Quintard H, Zerlauth JB, Maibach T, Carteron L, Suys T, et al: Bedside cerebral microdialysis monitoring of delayed cerebral hypoperfusion in comatose patients with poor grade aneurysmal subarachnoid haemorrhage. J Neurol Neurosurg Psychiatry 88:332-338, 2017

25. Ronne-Engström E, Cesarini KG, Enblad P, Hesselager G, Marklund N, Nilsson P, et al: Intracerebral microdialysis in neurointensive care: the use of urea as an endogenous reference compound. J Neurosurg 94:397-402, 2001

26. Rostami E, Engquist H, Howells T, Ronne-Engström E, Nilsson P, Hillered LT, et al: The correlation between cerebral blood flow measured by bedside xenon-CT and brain chemistry monitored by microdialysis in the acute phase following subarachnoid hemorrhage. Front Neurol 8:369, 2017

27. Ryttlefors M, Howells T, Nilsson P, Ronne-Engström E, Enblad P: Secondary insults in subarachnoid hemorrhage: occurrence and impact on outcome and clinical deterioration. Neurosurgery 61:704-715, 2007

28. Sanelli PC, Jou A, Gold R, Reichman M, Greenberg E, John $M$, et al: Using CT perfusion during the early baseline period in aneurysmal subarachnoid hemorrhage to assess for development of vasospasm. Neuroradiology 53:425-434, 2011

29. Sarrafzadeh AS, Haux D, Lüdemann L, Amthauer H, Plotkin M, Küchler I, et al: Cerebral ischemia in aneurysmal subarachnoid hemorrhage: a correlative microdialysis-PET study. Stroke 35:638-643, 2004

30. Sarrafzadeh AS, Sakowitz OW, Kiening KL, Benndorf G, Lanksch WR, Unterberg AW: Bedside microdialysis: a tool to monitor cerebral metabolism in subarachnoid hemorrhage patients? Crit Care Med 30:1062-1070, 2002
31. Schulz MK, Wang LP, Tange M, Bjerre P: Cerebral microdialysis monitoring: determination of normal and ischemic cerebral metabolisms in patients with aneurysmal subarachnoid hemorrhage. J Neurosurg 93:808-814, 2000

32. Steiner T, Juvela S, Unterberg A, Jung C, Forsting M, Rinkel $\mathrm{G}$ : European Stroke Organization guidelines for the management of intracranial aneurysms and subarachnoid haemorrhage. Cerebrovasc Dis 35:93-112, 2013

33. Vergouwen MD, Ilodigwe D, Macdonald RL: Cerebral infarction after subarachnoid hemorrhage contributes to poor outcome by vasospasm-dependent and -independent effects. Stroke 42:924-929, 2011

34. Yonas H, Darby JM, Marks EC, Durham SR, Maxwell C: CBF measured by Xe-CT: approach to analysis and normal values. J Cereb Blood Flow Metab 11:716-725, 1991

\section{Disclosures}

The authors report no conflict of interest concerning the materials or methods used in this study or the findings specified in this paper.

\section{Author Contributions}

Conception and design: all authors. Acquisition of data: Engquist. Analysis and interpretation of data: Engquist, Hillered, Enblad, Rostami. Drafting the article: Engquist, Enblad, Rostami. Critically revising the article: all authors. Reviewed submitted version of manuscript: all authors. Approved the final version of the manuscript on behalf of all authors: Engquist. Study supervision: Enblad, Rostami.

\section{Correspondence}

Henrik Engquist: Uppsala University Hospital, Uppsala, Sweden. henrik.engquist@akademiska.se. 